\title{
Editorial Statement for Mathematical Finance
}

\author{
Wing-Keung Wong ${ }^{1,2,3}$ (D) \\ 1 Department of Finance, Fintech Center, and Big Data Research Center, Asia University, Wufeng, \\ Taichung 41354, Taiwan; wong@asia.edu.tw \\ 2 Department of Medical Research, China Medical University Hospital, Taichung 40402, Taiwan \\ 3 Department of Economics and Finance, The Hang Seng University of Hong Kong, Hong Kong, China
}

Received: 7 January 2020; Accepted: 8 January 2020; Published: 21 January 2020

\begin{abstract}
Mathematics plays a vital role in many areas of finance and provides the theories and tools that have been widely used in all areas of finance. In this editorial, we tell authors the ideas on what types of papers we will accept for publication in the area of mathematical finance. We will discuss some well-cited papers of mathematical finance.
\end{abstract}

Keywords: mathematics; probability; statistics; finance; applications

Mathematics plays a vital role in many areas of finance. In particular, it provides the theories and tools that have been widely used in all areas of finance. Knowledge of mathematics, probability, statistics, and other analytic approaches is essential to develop methods and theories in finance and test their validity through the analysis of empirical real-world data. For example, mathematics, probability, and statistics could help to develop pricing models for financial assets such as equities, bonds, currencies, and derivative securities, and propose financially optimal strategies coherently to decision-makers according to their preferences. This section will bring together theory, practice, and applications of mathematical finance. We discuss some of the most cited papers, as follows:

Ly et al. (2019) develop the theory on both density and distribution functions for the quotient $Y=X_{1 /} X_{2}$ and the ratio of one variable over the sum of two variables $Z=X_{1 /}\left(X_{1}+X_{2}\right)$ of two dependent or independent random variables $X_{1}$ and $X_{2}$ by using copulas to capture the structures between $X_{1}$ and $X_{2}$, and extend the theory by establishing the density and distribution functions for the quotients $Y=X_{1 /} X_{2}$ and $Z=X_{1 /}\left(X_{1}+X_{2}\right)$ of two dependent normal random variables $X_{1}$ and $X_{2}$ in the case of Gaussian copulas. Thereafter, they develop the theory on the median for the ratios of both $Y$ and $Z$ on two normal random variables $X_{1}$ and $X_{2}$ and extend the result of the median for $Z$ to a larger family of symmetric distributions and symmetric copulas of $X_{1}$ and $X_{2}$. In addition, they introduce the Monte Carlo algorithm, numerical analysis, and graphical approach to efficiently compute the complicated integrals and study the behaviors of density and distribution and illustrate their proposed approaches by using a simulation study with ratios of normal random variables on several different copulas, including Gaussian, Student- $t$, Clayton, Gumbel, Frank, and Joe Copulas, and discuss the behaviors via all copulas above with the same Kendall's coefficient. They find that copulas make big impacts from different copulas on the behavior of distributions, especially on median, spread, scale, and skewness effects.

Golodnikov et al. (2019) show that CVaR linear regression can be reduced to minimize the Rockafellar error function with linear programming. They establish the theoretical basis for the analysis with the quadrangle theory of risk functions and derive relationships between elements of CVaR quadrangle and mixed-quantile quadrangle for discrete distributions with equally probable atoms. They present two equivalent variants of discretization of the integral, which resulted in two sets of parameters for the mixed-quantile quadrangle. For the first set of parameters, the minimization of error from the CVaR quadrangle is equivalent to the minimization of the Rockafellar error from the 
mixed-quantile quadrangle. Alternatively, a two-stage procedure based on the decomposition theorem can be used for CVaR linear regression with both sets of parameters. They find that this procedure is valid because the deviation in the mixed-quantile quadrangle (called mixed CVaR deviation) coincides with the deviation in the CVaR quadrangle for both sets of parameters. In addition, they illustrate theoretical results with a case study demonstrating the numerical efficiency of the suggested approach.

De Gaetano (2018) investigates the relevance of structural breaks for forecasting the volatility of daily returns on BRICS countries by using the data from 19 July 1999 to 16 July 2015 to identify structural breaks in the unconditional variance, a binary segmentation algorithm with a test. He introduces some forecast combinations that account for the identified structural breaks and evaluate and compare their performance by using the model confidence set (MCS). He obtains significant evidence of the relevance of the structural breaks, in particular, in the regimes identified by the structural breaks; a substantial change in the unconditional variance is quite evident. In addition, He finds that the combination that averages forecasts obtained using different rolling estimation windows outperforms all the other combinations.

Van Dijk et al. (2018) propose improved regression models to estimate calibrated parameters (including the market variables in a real-world simulation), predict out-of-sample implied volatility surfaces, and evaluate the impact on the solvency capital requirement for different points in time.

Korkmaz et al. (2018) introduce and study a new three-parameter Pareto distribution. They discuss various mathematical and statistical properties of the new model to perform some estimation methods of the model parameters, use the peaks-over-threshold method to estimate value-at-risk (VaR) by means of the proposed distribution, and compare the distribution with a few other models to show its versatility in modeling data with heavy tails. In addition, they present VaR estimation with the Burr $x$ Pareto distribution by using time series data and consider the new model as an alternative VaR model against the generalized Pareto model for financial institutions.

The popular replication formula to price variance swaps assumes continuity of traded option strikes. In practice, however, there is only a discrete set of option strikes traded on the market. Le Floc' $h$ (2018) presents different discrete replication strategies and explains why the continuous replication price is more relevant.

Ghitany et al. (2018) propose an alternative generalization of the Pareto distribution, study its properties, and apply their proposed model to analyze earthquake insurance data.

Nagy and Ormos (2018) introduce a spectral clustering-based method to show that stock prices contain not only firm but also network-level information. Clustering different stock indices and reconstructing the equity index graph from historical daily closing prices, they show that tail events have a minor effect on the equity index structure. In addition, they find that covariance and Shannon entropy do not provide enough information about the network, but Gaussian clusters can explain a substantial part of the total variance.

Employing a time-varying vector autoregression with stochastic volatility, Feldkircher and Huber (2018) compare the transmission of a conventional monetary policy shock with that of an unexpected decrease in the term spread, which mirrors quantitative easing. They find that the spread shock works mainly through a boost to consumer wealth growth, while a conventional monetary policy shock affects real output growth via a broad credit/bank lending channel. In addition, they find small output effects of a conventional monetary policy shock during the period of the global financial crisis and stronger effects in its aftermath. Their findings imply that when the central bank has left the policy rate unaltered for an extended period of time, a policy surprise might boost output particularly strongly while the spread shock has affected output growth most strongly during the period of the global financial crisis, and less so thereafter.

Funding: This research received no external funding.

Acknowledgments: The author would like to thank Robert B. Miller and Howard E. Thompson for their continuous guidance and encouragement. For financial and research support, the author acknowledges Asia University, China Medical University Hospital, The Hang Seng University of Hong Kong, the Research Grants Council 
of Hong Kong (project number 12500915), and Ministry of Science and Technology (MOST, Project Numbers 106-2410-H-468-002 and 107-2410-H-468-002-MY3), Taiwan.

Conflicts of Interest: The author declares no conflict of interest.

\section{References}

De Gaetano, Davide. 2018. Forecast Combinations for Structural Breaks in Volatility: Evidence from BRICS Countries. Journal Risk Financial Management 11: 64. [CrossRef]

Feldkircher, Martin, and Florian Huber. 2018. Unconventional U.S. Monetary Policy: New Tools, Same Channels? Journal Risk Financial Management 11: 71. [CrossRef]

Ghitany, Mohamed E., Emilio Gómez-Déniz, and Saralees Nadarajah. 2018. A New Generalization of the Pareto Distribution and Its Application to Insurance Data. Journal Risk Financial Management 11: 10. [CrossRef]

Golodnikov, Alex, Viktor Kuzmenko, and Stan Uryasev. 2019. CVaR Regression Based on the Relation between CVaR and Mixed-Quantile Quadrangles. Journal Risk Financial Management 12: 107. [CrossRef]

Korkmaz, Mustafa Ç, Emrah Altun, Haitham M. Yousof, Ahmed Z. Afify, and Saralees Nadarajah. 2018. The Burr X Pareto Distribution: Properties, Applications and VaR Estimation. Journal Risk Financial Management 11: 1. [CrossRef]

Le Floc'h, Fabien. 2018. Variance Swap Replication: Discrete or Continuous? Journal Risk Financial Management 11: 11. [CrossRef]

Ly, Sel, Kim-Hung Pho, Sal Ly, and Wing-Keung Wong. 2019. Determining Distribution for the Quotients of Dependent and Independent Random Variables by Using Copulas. Journal Risk Financial Management 12: 42. [CrossRef]

Nagy, László, and Mihály Ormos. 2018. Friendship of Stock Market Indices: A Cluster-Based Investigation of Stock Markets. Journal Risk Financial Management 11: 88. [CrossRef]

Van Dijk, Marcel T. P., Cornelis S. L. De Graaf, and Cornelis W. Oosterlee. 2018. Between P and @: The Peasure for Pricing in Asset Liability Management. Journal Risk Financial Management 11: 67. [CrossRef]

(C) 2020 by the author. Licensee MDPI, Basel, Switzerland. This article is an open access article distributed under the terms and conditions of the Creative Commons Attribution (CC BY) license (http://creativecommons.org/licenses/by/4.0/). 